\title{
The Dirichlet Problem for the 2D Laplace Equation in a Domain with Cracks without Compatibility Conditions at Tips of the Cracks
}

\author{
P. A. Krutitskii \\ KIAM, Miusskaya Square 4, Moscow 125047, Russia \\ Correspondence should be addressed to P. A. Krutitskii, krutitsk@mail.ru
}

Received 21 March 2012; Accepted 11 June 2012

Academic Editor: Vladimir Mityushev

Copyright (C) 2012 P. A. Krutitskii. This is an open access article distributed under the Creative Commons Attribution License, which permits unrestricted use, distribution, and reproduction in any medium, provided the original work is properly cited.

We study the Dirichlet problem for the 2D Laplace equation in a domain bounded by smooth closed curves and smooth cracks. In the formulation of the problem, we do not require compatibility conditions for Dirichlet's boundary data at the tips of the cracks. However, if boundary data satisfies the compatibility conditions at the tips of the cracks, then this is a particular case of our problem. The cases of both interior and exterior domains are considered. The wellposed formulation of the problem is given, theorems on existence and uniqueness of a classical solution are proved, and the integral representation for a solution is obtained. It is shown that weak solution of the problem does not typically exist, though the classical solution exists. The asymptotic formulae for singularities of a solution gradient at the tips of the cracks are presented.

\section{Introduction}

Boundary value problems in 2D domains with cracks (double-sided open arcs) are very important for applications [1].

It is known that if the Dirichlet problem for the Laplace equation is considered in a 2D domain bounded by sufficiently smooth closed curves, and if the function specified in the boundary condition is smooth enough, then existence of a classical solution follows from existence of a weak solution. In the present paper, we consider the Dirichlet problem for the Laplace equation in both interior and exterior 2D domain bounded by closed curves and double-sided open arcs (cracks) of an arbitrary shape. The Dirichlet condition is specified on the whole boundary, that is, on both closed curves and on the cracks, so that different functions may be specified on opposite sides of the cracks. The case of this problem, when the Dirichlet boundary data satisfies compatibility conditions at the tips of the cracks, 
has been previously studied in [2-6], where theorems on existence and uniqueness of a classical solution have been proved, and the integral representation for a classical solution has been obtained. In the present paper, this problem is considered in case when the Dirichlet boundary data may not satisfy the compatibility conditions at the tips of the cracks. However if boundary data satisfies the compatibility conditions at the tips of the cracks, then this is a particular case of our problem. We prove that there exists a unique classical solution to our problem and obtain an integral representation for the classical solution. In addition, we prove that a weak solution to our problem may not exist even if both cracks and functions in the boundary conditions are sufficiently smooth. This result follows from the fact that the square of the gradient of a classical solution, basically, is not integrable near the ends of the cracks, since singularities of the gradient are rather strong there. This result is very important for numerical analysis, it shows that finite elements and finite difference methods cannot be applied to numerical treatment of the Dirichlet problem in question directly, since all these methods imply existence of a weak solution. To use difference methods for numerical analysis, one has to localize all strong singularities first and next to use difference method in a domain excluding the neighbourhoods of the singularities. The asymptotic formulae for singularities of a solution gradient at the tips of the cracks are presented.

\section{Formulation of the Problem}

By an open curve we mean a simple smooth nonclosed arc of finite length without selfintersections [7].

Let $\Gamma$ be a set of curves, which may be both closed and open. We say that $\Gamma \in C^{2, \lambda}$ (or $\Gamma \in C^{1, \lambda}$ ) if curves $\Gamma$ belong to the class $C^{2, \lambda}$ (or $C^{1, \lambda}$ ) with the Hölder exponent $\lambda \in(0,1]$.

In a plane in Cartesian coordinates $x=\left(x_{1}, x_{2}\right) \in R^{2}$, we consider a multiply connected domain bounded by simple open curves $\Gamma_{1}^{1}, \ldots, \Gamma_{N_{1}}^{1} \in C^{2, \lambda}$ and simple closed curves $\Gamma_{1}^{2}, \ldots, \Gamma_{N_{2}}^{2} \in C^{2, \lambda}, \lambda \in(0,1]$, in such a way that all curves do not have common points, in particular, endpoints. We will consider both the case of an exterior domain and the case of an interior domain when the curve $\Gamma_{1}^{2}$ encloses all others. Set

$$
\Gamma^{1}=\bigcup_{n=1}^{N_{1}} \Gamma_{n}^{1}, \quad \Gamma^{2}=\bigcup_{n=1}^{N_{2}} \Gamma_{n}^{2}, \quad \Gamma=\Gamma^{1} \cup \Gamma^{2}
$$

The connected domain bounded by $\Gamma^{2}$ and containing curves $\Gamma^{1}$ will be called $\Phi$, so that $\partial \Phi=\Gamma^{2}, \Gamma^{1} \subset \Phi$. We assume that each curve $\Gamma_{n}^{j}$ is parametrized by the arc length $s$ :

$$
\Gamma_{n}^{j}=\left\{x: x=x(s)=\left(x_{1}(s), x_{2}(s)\right), s \in\left[a_{n}^{j}, b_{n}^{j}\right]\right\}, \quad n=1, \ldots, N_{j}, j=1,2,
$$

so that,

$$
a_{1}^{1}<b_{1}^{1}<\cdots<a_{N_{1}}^{1}<b_{N_{1}}^{1}<a_{1}^{2}<b_{1}^{2}<\cdots<a_{N_{2}}^{2}<b_{N_{2}}^{2}
$$


and the domain $\Phi$ is placed to the right when the parameter $s$ increases on $\Gamma_{n}^{2}$. The points $x \in \Gamma$ and values of the parameter $s$ are in one-to-one correspondence except the points $a_{n}^{2}$, $b_{n}^{2}$, which correspond to the same point $x$ for $n=1, \ldots, N_{2}$. Further on, the sets of the intervals

$$
\bigcup_{n=1}^{N_{1}}\left[a_{n}^{1}, b_{n}^{1}\right], \quad \bigcup_{n=1}^{N_{2}}\left[a_{n}^{2}, b_{n}^{2}\right], \quad \bigcup_{j=1}^{2} \bigcup_{n=1}^{N_{j}}\left[a_{n}^{j}, b_{n}^{j}\right]
$$

on the $O s$-axis will be denoted by $\Gamma^{1}, \Gamma^{2}$, and $\Gamma$ also.

Set $C^{0}\left(\Gamma_{n}^{2}\right)=\left\{\mathcal{F}(s): \mathcal{F}(s) \in C^{0}\left[a_{n}^{2}, b_{n}^{2}\right], \mathcal{F}\left(a_{n}^{2}\right)=\mathcal{F}\left(b_{n}^{2}\right)\right\}$, and $C^{0}\left(\Gamma^{2}\right)=\bigcap_{n=1}^{N_{2}} C^{0}\left(\Gamma_{n}^{2}\right)$. The tangent vector to $\Gamma$ in the point $x(s)$, in the direction of the increment of $s$, will be denoted by $\tau_{x}=(\cos \alpha(s), \sin \alpha(s))$, while the normal vector coinciding with $\tau_{x}$ after rotation through an angle of $\pi / 2$ in the counterclockwise direction will be denoted by $\mathbf{n}_{x}=(\sin \alpha(s),-\cos \alpha(s))$. According to the chosen parametrization $\cos \alpha(s)=x_{1}^{\prime}(s), \sin \alpha(s)=x_{2}^{\prime}(s)$. Thus, $\mathbf{n}_{x}$ is an inward normal to $\Phi$ on $\Gamma^{2}$. By $X$ we denote the point set consisting of the endpoints of $\Gamma^{1}$ : $X=\bigcup_{n=1}^{N_{1}}\left(x\left(a_{n}^{1}\right) \cup x\left(b_{n}^{1}\right)\right)$.

We consider $\Gamma^{1}$ as a set of cracks (or double-sided open arcs). The side of the crack $\Gamma^{1}$, which is situated on the left when the parameter $s$ increases, will be denoted by $\left(\Gamma^{1}\right)^{+}$, while the opposite side will be denoted by $\left(\Gamma^{1}\right)^{-}$.

We say that the function $u(x)$ belongs to the smoothness class $\mathbf{K}_{1}$, if

(1) $u \in C^{0}\left(\overline{\Phi \backslash \Gamma^{1}} \backslash X\right) \cap C^{2}\left(\Phi \backslash \Gamma^{1}\right), \quad \nabla u \in C^{0}\left(\overline{\Phi \backslash \Gamma^{1}} \backslash \Gamma^{2} \backslash X\right)$,

(2) in the neighbourhood of any point $x(d) \in X$, both the inequality

$$
|u(x)|<\text { Const }
$$

and the equality

$$
\lim _{r \rightarrow+0} \int_{\partial S(d, r)}\left|\frac{\partial u(x)}{\partial \mathbf{n}_{x}}\right| d l=0
$$

hold, where the curvilinear integral of the first kind is taken over a circumference $\partial S(d, r)$ of a radius $r$ with the center in the point $x(d)$, in addition, $\mathbf{n}_{x}$ is a normal in the point $x \in \partial S(d, r)$, directed to the center of the circumference and $d=a_{n}^{1}$ or $d=b_{n}^{1}, n=1, \ldots, N_{1}$.

Remark 2.1. By $C^{0}\left(\overline{\boldsymbol{\Phi} \backslash \Gamma^{1}} \backslash X\right)$ we denote the class of continuous in $\overline{\boldsymbol{\Phi}} \backslash \Gamma^{1}$ functions, which are continuously extensible to the sides of the cracks $\Gamma^{1} \backslash X$ from the left and from the right, but their limiting values on $\Gamma^{1} \backslash X$ can be different from the left and from the right, so that these functions may have a jump on $\Gamma^{1} \backslash X$. To obtain the definition of the class $C^{0}\left(\overline{\boldsymbol{\Phi} \backslash \Gamma^{1}} \backslash \Gamma^{2} \backslash X\right)$ we have to replace $C^{0}\left(\overline{\Phi \backslash \Gamma^{1}} \backslash X\right)$ by $C^{0}\left(\overline{\Phi \backslash \Gamma^{1}} \backslash \Gamma^{2} \backslash X\right)$ and $\bar{\Phi} \backslash \Gamma^{1}$ by $\Phi \backslash \Gamma^{1}$ in the previous sentence. 


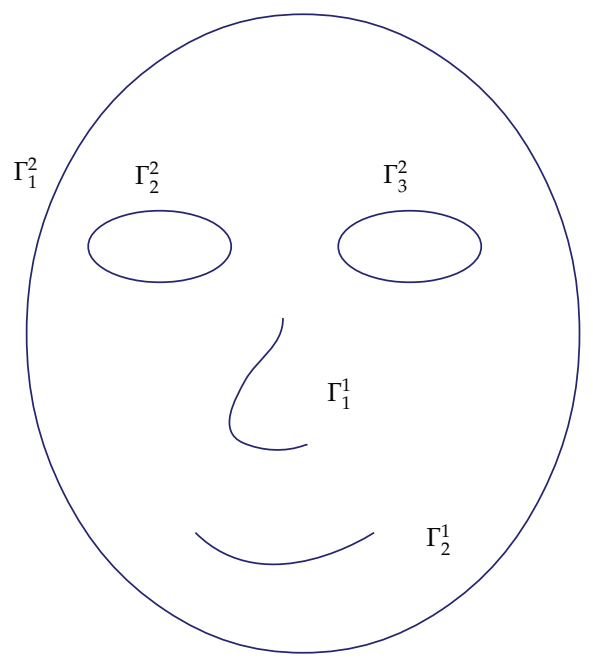

Figure 1: An example of an interior domain.

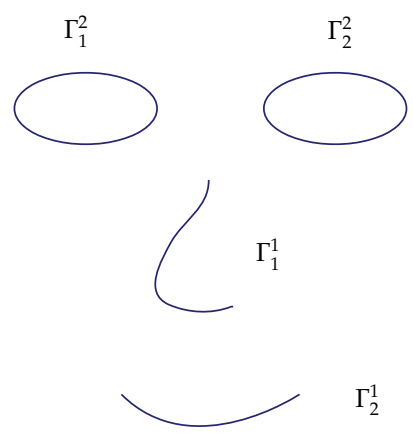

Figure 2: An example of an exterior domain.

Let us formulate the Dirichlet problem for the Laplace equation in a domain $\mathscr{\mathcal { }} \backslash \Gamma^{1}$ (interior or exterior, see Figures 1 and 2).

\section{Problem $\mathbf{D}_{1}$}

Find a function $u(x)$ from the class $\mathbf{K}_{1}$, so that $u(x)$ obeys the Laplace equation

$$
u_{x_{1} x_{1}}(x)+u_{x_{2} x_{2}}(x)=0
$$

in $\Phi \backslash \Gamma^{1}$ and satisfies the boundary conditions

$$
\left.u(x)\right|_{x(s) \in\left(\Gamma^{1}\right)^{+}}=F^{+}(s),\left.\quad u(x)\right|_{x(s) \in\left(\Gamma^{1}\right)^{-}}=F^{-}(s),\left.\quad u(x)\right|_{x(s) \in \Gamma^{2}}=F(s) .
$$


If $\Phi$ is an exterior domain, then we add the following condition at infinity:

$$
|u(x)| \leq \text { const }, \quad|x|=\sqrt{x_{1}^{2}+x_{2}^{2}} \longrightarrow \infty
$$

All conditions of the problem $\mathbf{D}_{1}$ must be satisfied in a classical sense. The boundary conditions (2.8) on $\Gamma^{1}$ must be satisfied in the interior points of $\Gamma^{1}$, their validity at the ends of $\Gamma^{1}$ is not required.

Theorem 2.2. If $\Gamma \in C^{2, \lambda}, \lambda \in(0,1]$, then there is no more than one solution to the problem $\mathbf{D}_{1}$.

Proof. It is sufficient to prove that the homogeneous problem $\mathbf{D}_{1}$ admits the trivial solution only. Let $u^{0}(x)$ be a solution to the homogeneous problem $\mathbf{D}_{1}$ with $F^{+}(s) \equiv F^{-}(s) \equiv 0, F(s) \equiv 0$. Let $S(d, \epsilon)$ be a disc of a small enough radius $\epsilon$ with the center in the point $x(d)\left(d=a_{n}^{1}\right.$ or $\left.d=b_{n}^{1}, n=1, \ldots, N_{1}\right)$. Let $\Gamma_{n, \epsilon}^{1}$ be a set consisting of such points of the curve $\Gamma_{n}^{1}$ which do not belong to $\operatorname{discs} S\left(a_{n}^{1}, \epsilon\right)$ and $S\left(b_{n}^{1}, \epsilon\right)$. We choose a number $\epsilon_{0}$ so small that the following conditions are satisfied:

(1) for any $0<\epsilon \leq \epsilon_{0}$ the set of points $\Gamma_{n, \epsilon}^{1}$ is a unique non-closed arc for each $n=1, \ldots, N_{1}$,

(2) the points belonging to $\Gamma \backslash \Gamma_{n}^{1}$ are placed outside the discs $S\left(a_{n}^{1}, \epsilon_{0}\right), S\left(b_{n}^{1}, \epsilon_{0}\right)$ for any $n=1, \ldots, N_{1}$,

(3) discs of radius $\epsilon_{0}$ with centers in different ends of $\Gamma^{1}$ do not intersect.

Set $\Gamma^{1, \epsilon}=\cup_{n=1}^{N_{1}} \Gamma_{n, \epsilon}^{1}, S_{\epsilon}=\left(\cup_{n=1}^{N_{1}}\left[S\left(a_{n}^{1}, \epsilon\right) \cup S\left(b_{n}^{1}, \epsilon\right)\right]\right), \Phi_{\epsilon}=\Phi \backslash \Gamma^{1, \epsilon} \backslash S_{\epsilon}$. If $\Phi$ is an exterior domain, then we set $\Phi_{\epsilon, R}=\Phi_{\epsilon} \cap S^{R}$, where $S^{R}$ is a disc with a center in the origin and with sufficiently large radius $R$.

Since $\Gamma^{2} \in C^{2, \lambda}, u^{0}(x) \in C^{0}\left(\bar{\Phi} \backslash \Gamma^{1}\right)$ (remind that $u^{0}(x) \in \mathbf{K}_{1}$ ), and since $\left.u^{0}\right|_{\Gamma^{2}}=0 \in$ $C^{2, \lambda}\left(\Gamma^{2}\right)$, and owing to the lemma on regularity of solutions of elliptic equations near the boundary [8, lemma 6.18], we obtain $u^{0}(x) \in C^{1}\left(\bar{\Phi} \backslash \Gamma^{1}\right)$. Since $u^{0}(x) \in \mathbf{K}_{1}$, we observe that $u^{0}(x) \in C^{1}\left(\bar{\Phi}_{\epsilon}\right)$ for any $\epsilon \in\left(0, \epsilon_{0}\right]$. By $C^{1}\left(\bar{\Phi}_{\epsilon}\right)$ we mean $C^{1}\left(\Phi_{\epsilon} \cup \Gamma^{2} \cup\left(\Gamma^{1, \epsilon}\right)^{+} \cup\left(\Gamma^{1, \epsilon}\right)^{-} \cup \partial S_{\epsilon}\right)$. Analogously, in case of exterior domain $\Phi: u^{0}(x) \in C^{1}\left(\bar{\Phi}_{\epsilon, R}\right)$ for $\epsilon \in\left(0, \epsilon_{0}\right]$. Let $\Phi$ be an interior domain. Since the boundary of a domain $\Phi_{\epsilon}$ is piecewise smooth, we write out Green's formula [9, page 328] for the function $u^{0}(x)$ :

$$
\begin{aligned}
\left\|\nabla u^{0}\right\|_{L_{2}\left(\Phi_{e}\right)}^{2}= & \int_{\Gamma^{1, e}}\left(u^{0}\right)^{+}\left(\frac{\partial u^{0}}{\partial \mathbf{n}_{x}}\right)^{+} d s-\int_{\Gamma^{1, e}}\left(u^{0}\right)^{-}\left(\frac{\partial u^{0}}{\partial \mathbf{n}_{x}}\right)^{-} d s \\
& -\int_{\Gamma^{2}} u^{0} \frac{\partial u^{0}}{\partial \mathbf{n}_{x}} d s+\int_{\partial S_{e}} u^{0} \frac{\partial u^{0}}{\partial \mathbf{n}_{x}} d l .
\end{aligned}
$$

By $\mathbf{n}_{x}$ the exterior (with respect to $\Phi_{\epsilon}$ ) normal on $\partial S_{\epsilon}$ at the point $x \in \partial S_{\epsilon}$ is denoted. By the superscripts + and - we denote the limiting values of functions on $\left(\Gamma^{1}\right)^{+}$and on 
$\left(\Gamma^{1}\right)^{-}$, respectively. Since $u^{0}(x)$ satisfies the homogeneous boundary condition (2.8) on $\Gamma$, we observe that $\left.u^{0}\right|_{\Gamma^{2}}=0$ and $\left.\left(u^{0}\right)^{ \pm}\right|_{\Gamma^{1, \epsilon}}=0$ for any $\epsilon \in\left(0, \epsilon_{0}\right]$. Therefore, identity (2.10) takes the form

$$
\left\|\nabla u^{0}\right\|_{L_{2}\left(\Phi_{e}\right)}^{2}=\int_{\partial S_{e}} u^{0} \frac{\partial u^{0}}{\partial \mathbf{n}_{x}} d l, \quad \epsilon \in\left(0, \epsilon_{0}\right] .
$$

Setting $\epsilon \rightarrow+0$ in (2.11), taking into account that $u^{0}(x) \in \mathbf{K}_{1}$ and using the relationships (2.6), (2.5) we obtain

$$
\left\|\nabla u^{0}\right\|_{L_{2}\left(\Phi \mid \Gamma^{1}\right)}^{2}=\lim _{\epsilon \rightarrow+0}\left\|\nabla u^{0}\right\|_{L_{2}\left(\Phi_{\epsilon}\right)}^{2}=0 .
$$

From the homogeneous boundary conditions (2.8) we conclude that $u^{0}(x) \equiv 0$ in $\Phi \backslash \Gamma^{1}$, where $\Phi$ is an interior domain.

Let $\Phi$ be an exterior domain. Since the boundary of a domain $\Phi_{\epsilon, R}$ is piecewise smooth and since $u^{0}(x) \in C^{1}\left(\bar{\Phi}_{\epsilon, R}\right)$ for any $\epsilon \in\left(0, \epsilon_{0}\right]$, we may write Green's formula in a domain $\Phi_{\epsilon, R}$ for a harmonic function $u^{0}(x)$ [9, c.328]:

$$
\begin{aligned}
\left\|\nabla u^{0}\right\|_{L_{2}\left(\Phi_{e, R}\right)}^{2}= & \int_{\Gamma^{1, e}}\left(u^{0}\right)^{+}\left(\frac{\partial u^{0}}{\partial \mathbf{n}_{x}}\right)^{+} d s-\int_{\Gamma^{1, e}}\left(u^{0}\right)^{-}\left(\frac{\partial u^{0}}{\partial \mathbf{n}_{x}}\right)^{-} d s \\
& -\int_{\Gamma^{2}} u^{0} \frac{\partial u^{0}}{\partial \mathbf{n}_{x}} d s+\int_{\partial S_{e}} u^{0} \frac{\partial u^{0}}{\partial \mathbf{n}_{x}} d l+\int_{\partial S^{R}} u^{0} \frac{\partial u^{0}}{\partial|x|} d l .
\end{aligned}
$$

By $\mathbf{n}_{x}$ on $\partial S_{\epsilon}$ we denote an outward (with respect to $\Phi_{\epsilon, R}$ ) normal in the point $x \in \partial S_{\epsilon}$. It follows from condition (2.9) and from the theorem on behaviour of a gradient of a harmonic function at infinity [9, page 373] that

$$
\frac{\partial u^{0}(x)}{\partial|x|}=O\left(\frac{1}{|x|^{2}}\right), \quad \text { as }|x| \longrightarrow \infty .
$$

Consequently,

$$
\lim _{R \rightarrow \infty} \int_{\partial S^{R}} u^{0}(x) \frac{\partial u^{0}(x)}{\partial|x|} d l=0
$$

and formula (2.13) transforms to the formula (2.10) as $R \rightarrow \infty$. Repeating all arguments, presented above for the case of an interior domain $\Phi$, we arrive to formula (2.12). Taking into account homogeneous boundary conditions (2.8), we obtain from (2.12) that $u^{0}(x) \equiv 0$ in $\boldsymbol{\Phi} \backslash \Gamma^{1}$, where $\Phi$ is an exterior domain. Thus, in all cases $u^{0}(x) \equiv 0$ in $\boldsymbol{\Phi} \backslash \Gamma^{1}$. Theorem is proved.

Remark 2.3. The maximum principle cannot be used for the proof of the theorem even in the case of an interior domain $\Phi$, since the solution to the problem may not satisfy the boundary 
condition (2.8) at the ends of the cracks, and it may not be continuous at the ends of the cracks.

\section{Properties of the Double Layer Potential on the Open Curve}

Let $\gamma$ be an open curve of class $C^{1, \lambda}, \lambda \in(0,1]$. Assume that $\gamma$ is parametrized by the arc length $s: \gamma=\left\{x: x(s)=\left(x_{1}(s), x_{2}(s)\right), s \in[a, b]\right\}$. The points $x \in \Gamma$ and values of the parameter $s$ are in one-to-one correspondence, so the segment $[a, b]$ will be also denoted by $\gamma$. The tangent vector to $\gamma$ in the point $x(s)$, in the direction of the increment of $s$, will be denoted by $\tau_{x}=(\cos \alpha(s)$, $\sin \alpha(s))$, while the normal vector to $\gamma$ in the point $x(s)$ will be denoted by $\mathbf{n}_{x}=(\sin \alpha(s),-\cos \alpha(s))$. According to the chosen parametrization $\cos \alpha(s)=x_{1}^{\prime}(s), \sin \alpha(s)=x_{2}^{\prime}(s)$. The side of the crack $\gamma$, which is situated on the left when the parameter $s$ increases, will be denoted by $\gamma^{+}$, while the opposite side will be denoted by $\gamma^{-}$. Let $X_{\gamma}=x(a) \cup x(b)$ be a set of the ends of $\gamma$. plane

Set $\mu(s) \in C^{0, \lambda}[a, b]$, and consider the double layer potential for Laplace equation in a

$$
\mathcal{W}[\mu](x)=-\frac{1}{2 \pi} \int_{a}^{b} \mu(\sigma) \frac{\partial}{\partial \mathbf{n}_{y}} \ln |x-y(\sigma)| d \sigma .
$$

Set $z=x_{1}+i x_{2}, t=t(\sigma)=\left(y_{1}(\sigma)+i y_{2}(\sigma)\right) \in \gamma, \widehat{\mu}(t)=\widehat{\mu}(t(\sigma))=\mu(\sigma)$. If $\mu(s) \in C^{0, \lambda}[a, b]$, then $\widehat{\mu}(t) \in C^{0, \lambda}(\gamma)$, since

$$
\begin{aligned}
\left|\widehat{\mu}\left(t_{2}\right)-\widehat{\mu}\left(t_{1}\right)\right| & =\left|\widehat{\mu}\left(t\left(\sigma_{2}\right)\right)-\widehat{\mu}\left(t\left(\sigma_{1}\right)\right)\right|=\left|\mu\left(\sigma_{2}\right)-\mu\left(\sigma_{1}\right)\right| \leq c\left|\sigma_{2}-\sigma_{1}\right|^{\lambda} \\
& =c\left(\frac{\left|\sigma_{2}-\sigma_{1}\right|}{\left|t\left(\sigma_{2}\right)-t\left(\sigma_{1}\right)\right|}\right)^{\lambda}\left|t\left(\sigma_{2}\right)-t\left(\sigma_{1}\right)\right|^{\lambda}=c \cdot c_{0}^{\lambda}\left|t_{2}-t_{1}\right|^{\lambda}
\end{aligned}
$$

where $c$ and $c_{0}$ are constants, $t_{2}=t\left(\sigma_{2}\right) \in \gamma, t_{1}=t\left(\sigma_{1}\right) \in \gamma$. We took into account in deriving the latter inequality that

$$
\frac{\left|\sigma_{2}-\sigma_{1}\right|}{\left|t\left(\sigma_{2}\right)-t\left(\sigma_{1}\right)\right|} \in C^{0}([a, b] \times[a, b])
$$

(see lemma 1 in [10]), whence

$$
\frac{\left|\sigma_{2}-\sigma_{1}\right|}{\left|t\left(\sigma_{2}\right)-t\left(\sigma_{1}\right)\right|} \leq c_{0}
$$

Consider the integral of the Cauchy type with the real density $\widehat{\mu}(t)$ :

$$
\Phi(z)=\frac{1}{2 \pi i} \int_{\gamma} \widehat{\mu}(t) \frac{d t}{t-z}
$$

then $\mathcal{W}[\mu](x)=-\operatorname{Re} \Phi(z)$. It follows from properties of the Cauchy type integral that if $\mu(\sigma) \in C^{0, \lambda}[a, b]$, then $\mathcal{W}[\mu](x) \in C^{0}\left(\overline{R^{2} \backslash \gamma} \backslash X_{\gamma}\right)$. This means that the potential $\mathcal{W}[\mu](x)$ 
is continuously extensible to $\gamma$ from the left and from the right in interior points (though its values on $\gamma$ from the left and from the right may be different). If, in addition, $\mu(d)=0$, then the potential $\mathcal{W}[\mu](x)$ is continuously extensible to the end $x(d)$, where $d=a$ or $d=b$ (see [7, Section 15.2]). Set

$$
\cos \psi(x, y)=\frac{x_{1}-y_{1}}{|x-y|}=-|x-y|_{y_{1},}^{\prime} \quad \sin \psi(x, y)=\frac{x_{2}-y_{2}}{|x-y|}=-|x-y|_{y_{2}}^{\prime}
$$

then $\psi(x, y)$ is a polar angle of the coordinate system with the origin in the point $y$. Formulae for $\cos \psi(x, y)$, $\sin \psi(x, y)$ define the angle $\psi(x, y)$ with indeterminacy up to $2 \pi m$ ( $m$ is an integer number). Let $S(d, \epsilon)$ be a disc of a sufficiently small radius $\epsilon$ with the center in $x(d)$ $(d=a$ or $d=b)$. From asymptotic formulae describing behavior of $\Phi(z)$ at the ends of $\gamma[7$, Section 22], we may derive the asymptotic formulae for $\mathcal{W}[\mu](x)=-\operatorname{Re} \Phi(z)$ at the ends of $\gamma$. Namely, for any $x \in S(d, \epsilon)$ and $x \notin \gamma$, the formula holds:

$$
\mathcal{W}[\mu](x)=\frac{ \pm \mu(d)}{2 \pi} \psi(x, x(d))+\Omega(x)
$$

Here by $\psi(x, x(d))$ we mean some fixed branch of this function, so that the branch varies continuously in $x$ in a neighbourhood of the point $x(d)$, cut along $\gamma$. The upper sign is taken if $d=a$, while the lower sign is taken if $d=b$. The function $\Omega(x)$ is continuous as $x \rightarrow x(d)$. Moreover, $\Omega(x)$ is continuous in $S(d, \epsilon)$ outside $\gamma$ and is continuously extensible from the left and from the right to the part of $\gamma$ lying in $S(d, \epsilon)$. It follows from formula (3.7) that for any $x \in S(d, \epsilon)$ and $x \notin \gamma$ the inequality holds

$$
|\mathcal{W}[\mu](x)| \leq \text { const. }
$$

Now we will study properties of derivatives of the double layer potential. It follows from Cauchy-Riemann relations that

$$
\frac{d \Phi(z)}{d z}=(\operatorname{Re} \Phi)_{x_{1}}^{\prime}-i(\operatorname{Re} \Phi)_{x_{2}}^{\prime}=-\mathcal{W}_{x_{1}}^{\prime}+i \mathcal{W}_{x_{2}}^{\prime}
$$

On the other hand, if $\mu(\sigma) \in C^{1, \lambda}[a, b]$, then for $z \notin \gamma$ :

$$
\begin{aligned}
\frac{d \Phi(z)}{d z} & =\frac{1}{2 \pi i} \int_{\gamma} \widehat{\mu}(t) \frac{d}{d z} \frac{1}{t-z} d t=-\frac{1}{2 \pi i} \int_{\gamma} \widehat{\mu}(t)\left(\frac{d}{d t} \frac{1}{t-z}\right) d t \\
& =-\frac{1}{2 \pi i}\left(\frac{\mu(b)}{t(b)-z}-\frac{\mu(a)}{t(a)-z}-\int_{\gamma} \frac{\widehat{\mu}^{\prime}(t)}{t-z} d t\right)
\end{aligned}
$$

where

$$
\frac{d \widehat{\mu}(t)}{d t}=\frac{d \mu(\sigma)}{d \sigma} \frac{d \sigma}{d t}=\frac{\mu^{\prime}(\sigma)}{t^{\prime}(\sigma)}=e^{-i \alpha(\sigma)} \mu^{\prime}(\sigma)
$$


Since $\gamma \in C^{1, \lambda}$, then $e^{-i \alpha(\sigma)} \in C^{0, \lambda}[a, b]$, so one can show that $\widehat{\mu}^{\prime}(t) \in C^{0, \lambda}(\gamma)$ (the proof repeats the given above proof of the fact that $\widehat{\mu}(t) \in C^{0, \lambda}(\gamma)$, if $\left.\mu(\sigma) \in C^{0, \lambda}[a, b]\right)$. From (3.9) and (3.10) and from properties of the Cauchy type integral [7, Section 15], it follows that if $\mu(\sigma) \in C^{1, \lambda}[a, b]$, then $\nabla \mathcal{W}[\mu](x) \in C^{0}\left(\overline{R^{2} \backslash \gamma} \backslash X_{\gamma}\right)$, that is, $\nabla \mathcal{W}[\mu](x)$ is continuously extensible to $\gamma$ from the left and from the right in interior points, though the limiting values of $\nabla \mathcal{W}[\mu](x)$ on $\gamma$ from the left and from the right can be different. We can write (3.10) in the form

$$
\frac{d \Phi(z)}{d z}=\frac{1}{2 \pi i}\left(\frac{\mu(b) e^{-i \psi(x, x(b))}}{|x-x(b)|}-\frac{\mu(a) e^{-i \psi(x, x(a))}}{|x-x(a)|}\right)+\Omega_{0}(z),
$$

where

$$
\Omega_{0}(z)=\frac{1}{2 \pi i} \int_{\gamma} \frac{\widehat{\mu}^{\prime}(t)}{t-z} d t
$$

It follows from $[7, \S 22]$ that for all $z \in S(d, \epsilon)(d=a$ or $d=b)$, such that $z \notin \gamma$, the inequality holds

$$
\left|\Omega_{0}(z)\right| \leq c_{0}\left(\left|\mu^{\prime}(d)\right| \ln \frac{1}{|x-x(d)|}+1\right) \leq c \ln \frac{1}{|x-x(d)|},
$$

where $c_{0}$ and $c$ are constants.

Comparing formulae (3.9) and (3.12), we obtain that for $x \in S(d, \epsilon)$ and $x \notin \gamma$, the formulae hold

$$
\begin{aligned}
& \frac{\partial \mathcal{W}[\mu](x)}{\partial x_{1}}=\frac{1}{2 \pi} \frac{\mp \mu(d)}{|x-x(d)|} \sin \psi(x, x(d))+\Omega_{1}(x), \\
& \frac{\partial \mathcal{W}[\mu](x)}{\partial x_{2}}=\frac{1}{2 \pi} \frac{ \pm \mu(d)}{|x-x(d)|} \cos \psi(x, x(d))+\Omega_{2}(x),
\end{aligned}
$$

where

$$
\left|\Omega_{j}(x)\right| \leq c_{1}\left(\left|\mu^{\prime}(d)\right| \ln \frac{1}{|x-x(d)|}+1\right) \leq c_{2} \ln \frac{1}{|x-x(d)|}, \quad j=1,2,
$$

$c_{1}, c_{2}$ are constants. The upper sign in formulae is taken if $d=a$, while the lower sign is taken if $d=b$. It follows from [7, Section 15.2] that if $\mu^{\prime}(d)=0$, then the functions $\Omega_{1}(x)$ and $\Omega_{2}(x)$ 
are continuously extensible to the end $x(d)$. Moreover, if $x \in S(d, \epsilon)$ and $x \notin \gamma$, then for the functions $\Omega_{1}(x)$ and $\Omega_{2}(x)$, the formulae hold:

$$
\begin{aligned}
\Omega_{1}(x) & =-\operatorname{Re} \Omega_{0}(z) \\
& =\mp \frac{\mu^{\prime}(d)}{2 \pi}\{\sin (\alpha(d)) \ln |x-x(d)|-\cos (\alpha(d)) \psi(x, x(d))\}+\Omega_{10}(x), \\
\Omega_{2}(x) & =\operatorname{Im} \Omega_{0}(z) \\
& = \pm \frac{\mu^{\prime}(d)}{2 \pi}\{\cos (\alpha(d)) \ln |x-x(d)|+\sin (\alpha(d)) \psi(x, x(d))\}+\Omega_{20}(x),
\end{aligned}
$$

which can be derived, using the asymptotics for $\Omega_{0}(z)$ from [7, § 22]. The upper sign in formulae is taken if $d=a$, while the lower sign is taken if $d=b$. Functions $\Omega_{10}(x)$ and $\Omega_{20}(x)$ are continuously extensible to the end $x(d)$. By $\psi(x, x(d))$ we mean some fixed branch of this function, which varies continuously in $x$ in a neighbourhood of a point $x(d)$, cut along $\gamma$.

Let $\mu(\sigma) \in C^{1, \lambda}[a, b]$, and let $\mathbf{n}_{x}$ be a normal in the point $x \in \partial S(d, \epsilon)$, directed to the center of the circumference $\partial S(d, \epsilon)$, that is, $\mathbf{n}_{x}=(-\cos \psi(x, x(d)),-\sin \psi(x, x(d)))$, then we obtain from (3.15) for $x \notin \gamma$

$$
\left.\frac{\partial \mathcal{W}[\mu](x)}{\partial \mathbf{n}_{x}}\right|_{\partial S(d, \epsilon)}=-\Omega_{1}(x) \cos \psi(x, x(d))-\Omega_{2}(x) \sin \psi(x, x(d)) .
$$

Therefore, according to (3.16):

$$
\left.\left|\frac{\partial \mathcal{W}[\mu](x)}{\partial \mathbf{n}_{x}}\right|\right|_{\partial S(d, \epsilon)} \leq \text { const }\left.\ln \frac{1}{|x-x(d)|}\right|_{\partial S(d, \epsilon)}=\text { const } \ln \frac{1}{\epsilon}
$$

since $|x-x(d)|=\epsilon$ on $\partial S(d, \epsilon)$. From here we obtain that

$$
\int_{\partial S(d, \epsilon)}\left|\frac{\partial \mathcal{W}[\mu](x)}{\partial \mathbf{n}_{x}}\right| d l=\int_{0}^{2 \pi}\left|\frac{\partial \mathcal{W}[\mu](x)}{\partial \mathbf{n}_{x}}\right| \epsilon d \psi \leq 2 \pi \text { const } \epsilon \ln \frac{1}{\epsilon} \longrightarrow 0
$$

if $\epsilon \rightarrow+0$, so

$$
\lim _{\epsilon \rightarrow+0} \int_{\partial S(d, \epsilon)}\left|\frac{\partial \mathcal{W}[\mu](x)}{\partial \mathbf{n}_{x}}\right| d l=0
$$


Now let $\epsilon$ be a fixed positive number (sufficiently small). Using formulae (3.15) and setting $r=|x-x(d)|, \psi=\psi(x, x(d))$, we consider the integral over the disc $S(d, \epsilon)$ :

$$
\begin{gathered}
\int_{S(d, \epsilon)}|\nabla \mathcal{W}[\mu](x)|^{2} d x=\int_{0}^{2 \pi} \int_{0}^{\epsilon}\left\{\left(\frac{\mu(d)}{2 \pi r}\right)^{2}+\frac{\mu(d)}{\pi r}\left(\mp \Omega_{1}(x) \sin \psi \pm \Omega_{2}(x) \cos \psi\right)\right. \\
\left.+\Omega_{1}^{2}(x)+\Omega_{2}^{2}(x)\right\} r d r d \psi=I_{1}+I_{2} \\
I_{1}=\frac{1}{2 \pi} \int_{0}^{\epsilon} \frac{1}{r} \mu^{2}(d) d r \\
I_{2}=\int_{0}^{2 \pi} \int_{0}^{\epsilon}\left\{\frac{\mu(d)}{\pi}\left(\mp \Omega_{1}(x) \sin \psi \pm \Omega_{2}(x) \cos \psi\right)+r\left(\Omega_{1}^{2}(x)+\Omega_{2}^{2}(x)\right)\right\} d r d \psi .
\end{gathered}
$$

The integral $I_{2}$ converges according to estimates (3.16):

$$
\left|I_{2}\right| \leq 4 c_{2} \int_{0}^{\epsilon} \ln \frac{1}{r}\left(|\mu(d)|+c_{2} \pi r \ln \frac{1}{r}\right) d r \leq \text { const. }
$$

Hence, if integral (3.22) converges, then the integral $I_{1}$ converges as well (as a difference of two convergent integrals), but the integral $I_{1}$ converges if and only if $\mu(d)=0$, while in other cases $I_{1}$ diverges. Thus, the integral (3.22) converges if and only if $\mu(d)=0$. Consequently $|\nabla \mathcal{W}[\mu](x)|$ belongs to $L_{2}(S(d, \epsilon))$ with small $\epsilon>0$ if and only if $\mu(d)=0$. Let us formulate obtained results in the form of the theorem.

Theorem 3.1. Let $\gamma$ be an open curve of class $C^{1, \lambda}, \lambda \in(0,1]$. Let $S(d, \epsilon)$ be a disc of a sufficiently small radius $\epsilon$ with the center in the point $x(d) \in X_{Y}(d=a$ or $d=b)$.

(i) If $\mu(s) \in C^{0, \lambda}[a, b]$, then $\mathcal{W}[\mu](x) \in C^{0}\left(\overline{R^{2} \backslash \gamma} \backslash X_{\gamma}\right)$ and for any $x \in S(d, \epsilon)$, such that $x \notin \gamma$, the relationships (3.7) and (3.8) hold.

(ii) If $\mu(s) \in C^{1, \lambda}[a, b]$, then

(1) $\nabla \mathcal{W}[\mu](x) \in C^{0}\left(\overline{R^{2} \backslash \gamma} \backslash X_{\gamma}\right)$;

(2) for any $x \in S(d, e)$, such that $x \notin \gamma$, the formulae (3.15) hold, in which the functions $\Omega_{1}(x)$ and $\Omega_{2}(x)$ satisfy relationships (3.16) and (3.17);

(3) for $\mathcal{W}[\mu](x)$ the property (3.21) holds;

(4) $|\nabla \mathcal{W}[\mu](x)|$ belongs to $L_{2}(S(d, \epsilon))$ for sufficiently small $\epsilon>0$ if and only if $\mu(d)=0$.

Remark 3.2. Each function of class $C^{0}\left(\overline{R^{2} \backslash \gamma} \backslash X_{\gamma}\right)$ is continuous in $R^{2} \backslash \gamma$, is continuously extensible to $\gamma \backslash X_{\gamma}$ from the left and from the right, but limiting values of such a function on $r \backslash X_{\gamma}$ from the left and from the right can be different, that is, the function may have a jump on $r \backslash X_{r}$.

Let us study smoothness of the direct value of the double layer potential on the open curve. 
Lemma 3.3. Let $\gamma$ be an open curve of class $C^{2, \lambda}, \lambda \in(0,1]$, and let $\mu(s) \in C^{0}[a, b]$. Let

$$
I_{1}(s)=-\frac{1}{2 \pi} \int_{\gamma} \mu(\sigma) \frac{\partial \ln |x(s)-y(\sigma)|}{\partial \mathbf{n}_{y}} d \sigma
$$

be the direct value of the double layer potential $w[\mu](x)$ on $\gamma$. Then

$$
I_{1}(s) \in C^{1, \lambda / 4}[a, b] .
$$

Proof. Let us prove that $I_{1}(s) \in C^{1, \lambda / 4}[a, b]$. Taking into account that $\mathbf{n}_{y}=\left(y_{2}^{\prime}(\sigma),-y_{1}^{\prime}(\sigma)\right)$, we find

$$
\begin{gathered}
\frac{\partial \ln |x(s)-y(\sigma)|}{\partial \mathbf{n}_{y}}=\frac{T(s, \sigma)}{g(s, \sigma)}, \quad g(s, \sigma)=\frac{|x(s)-y(\sigma)|^{2}}{(s-\sigma)^{2}}, \\
T(s, \sigma)=\frac{\left[x_{2}(s)-y_{2}(\sigma)\right] y_{1}^{\prime}(\sigma)-\left[x_{1}(s)-y_{1}(\sigma)\right] y_{2}^{\prime}(\sigma)}{(s-\sigma)^{2}} .
\end{gathered}
$$

Note that $y(\sigma)$ is a point on $\Gamma$ corresponding to $s=\sigma$. So, we may put $x(\sigma)=y(\sigma)$. For $j=1,2$, we have $[10, \S 3]$

$$
x_{j}(s)-x_{j}(\sigma)=(s-\sigma) Z_{j}^{1}(s, \sigma)=-x_{j}^{\prime}(\sigma)(\sigma-s)+(\sigma-s)^{2} Z_{j}^{2}(\sigma, s),
$$

where

$$
\begin{aligned}
& Z_{j}^{1}(s, \sigma)=\int_{0}^{1} x_{j}^{\prime}(\sigma+\xi(s-\sigma)) d \xi \in C^{1, \lambda}([a, b] \times[a, b]) \\
& Z_{j}^{2}(\sigma, s)=\int_{0}^{1} \xi x_{j}^{\prime \prime}(s+\xi(\sigma-s)) d \xi \in C^{0, \lambda}([a, b] \times[a, b]) .
\end{aligned}
$$

Note that the function

$$
g(s, \sigma)=\frac{|x(s)-x(\sigma)|^{2}}{(s-\sigma)^{2}}=\left\{\left[Z_{1}^{1}(s, \sigma)\right]^{2}+\left[Z_{2}^{1}(s, \sigma)\right]^{2}\right\} \in C^{1, \lambda}([a, b] \times[a, b])
$$

does not equal zero anywhere on $\Gamma$ and $g(s, s)=1$; therefore,

$$
\frac{1}{g(s, \sigma)} \in C^{1}([a, b] \times[a, b])
$$


Further,

$$
\begin{aligned}
\frac{\partial}{\partial s} \frac{1}{g(s, \sigma)} & =\frac{\partial}{\partial s} \frac{(s-\sigma)^{2}}{|x(s)-x(\sigma)|^{2}}=-\frac{g_{s}^{\prime}(s, \sigma)}{g^{2}(s, \sigma)} \\
& =-2 \frac{Z_{1}^{1}(s, \sigma)\left[Z_{1}^{1}(s, \sigma)\right]_{s}^{\prime}+Z_{2}^{1}(s, \sigma)\left[Z_{2}^{1}(s, \sigma)\right]_{s}^{\prime}}{g^{2}(s, \sigma)} \in C^{0, \lambda}([a, b] \times[a, b]) .
\end{aligned}
$$

Consequently, $1 / g(s, \sigma) \in C^{1, \lambda}([a, b] \times[a, b])$. Similarly,

$$
\begin{aligned}
T(s, \sigma) & =\frac{\left[x_{2}(s)-x_{2}(\sigma)\right] x_{1}^{\prime}(\sigma)-\left[x_{1}(s)-x_{1}(\sigma)\right] x_{2}^{\prime}(\sigma)}{(s-\sigma)^{2}} \\
& =\left[Z_{2}^{2}(\sigma, s) x_{1}^{\prime}(\sigma)-Z_{1}^{2}(\sigma, s) x_{2}^{\prime}(\sigma)\right] \in C^{0, \lambda}([a, b] \times[a, b]) .
\end{aligned}
$$

Consider $\partial T(s, \sigma) / \partial s=J_{1}(s, \sigma)-2 J_{2}(s, \sigma)$, where

$$
\begin{aligned}
J_{1}(s, \sigma) & =\frac{x_{2}^{\prime}(s) x_{1}^{\prime}(\sigma)-x_{1}^{\prime}(s) x_{2}^{\prime}(\sigma)}{(s-\sigma)^{2}} \\
& =\frac{\left[x_{2}^{\prime}(s)-x_{2}^{\prime}(\sigma)\right] x_{1}^{\prime}(\sigma)-\left[x_{1}^{\prime}(s)-x_{1}^{\prime}(\sigma)\right] x_{2}^{\prime}(\sigma)}{(s-\sigma)^{2}} \\
& =\frac{1}{s-\sigma}\left\{x_{1}^{\prime}(\sigma) \int_{0}^{1} x_{2}^{\prime \prime}(s+\xi(\sigma-s)) d \xi-x_{2}^{\prime}(\sigma) \int_{0}^{1} x_{1}^{\prime \prime}(s+\xi(\sigma-s)) d \xi\right\} \\
J_{2}(s, \sigma) & =\frac{\left[x_{2}(s)-x_{2}(\sigma)\right] x_{1}^{\prime}(\sigma)-\left[x_{1}(s)-x_{1}(\sigma)\right] x_{2}^{\prime}(\sigma)}{(s-\sigma)^{3}} \\
& =\frac{1}{s-\sigma}\left\{x_{1}^{\prime}(\sigma) \int_{0}^{1} \xi x_{2}^{\prime \prime}(s+\xi(\sigma-s)) d \xi-x_{2}^{\prime}(\sigma) \int_{0}^{1} \xi x_{1}^{\prime \prime}(s+\xi(\sigma-s)) d \xi\right\} .
\end{aligned}
$$

Then

$$
\begin{aligned}
\frac{\partial T(s, \sigma)}{\partial s} & =\frac{1}{s-\sigma}\left\{x_{1}^{\prime}(\sigma) \int_{0}^{1}(1-2 \xi) x_{2}^{\prime \prime}(s+\xi(\sigma-s)) d \xi-x_{2}^{\prime}(\sigma) \int_{0}^{1}(1-2 \xi) x_{1}^{\prime \prime}(s+\xi(\sigma-s)) d \xi\right\} \\
& =\frac{K(s, \sigma)}{s-\sigma}
\end{aligned}
$$


where $K(s, \sigma) \in C^{0, \lambda}([a, b] \times[a, b])$ and $K(s, s)=0$. According to [7, Section 5.7], the following representation holds

$$
\frac{\partial T(s, \sigma)}{\partial s}=\frac{K^{*}(s, \sigma)}{|s-\sigma|^{1-\lambda / 4}}
$$

$K^{*}(s, \sigma) \in C^{0,3 \lambda / 4}([a, b] \times[a, b])$. Using properties of Hölder functions [7], we obtain the representation

$$
\begin{aligned}
\frac{\partial}{\partial s} \frac{\partial \ln |x(s)-y(\sigma)|}{\partial \mathbf{n}_{y}} & =\frac{1}{g(s, \sigma)} \frac{\partial T(s, \sigma)}{\partial s}+T(s, \sigma) \frac{\partial}{\partial s} \frac{1}{g(s, \sigma)} \\
& =\frac{K_{1}(s, \sigma)}{|s-\sigma|^{1-\lambda / 4}}+K_{2}(s, \sigma),
\end{aligned}
$$

where $K_{1}(s, \sigma) \in C^{0,3 \lambda / 4}([a, b] \times[a, b]), K_{2}(s, \sigma) \in C^{0, \lambda}([a, b] \times[a, b])$. By formal differentiation under the integral, we find

$$
\begin{aligned}
\frac{d I_{1}(s)}{d s} & =-\frac{1}{2 \pi} \int_{\gamma} \mu(\sigma) \frac{\partial}{\partial s} \frac{\partial \ln |x(s)-y(\sigma)|}{\partial \mathbf{n}_{y}} d \sigma \\
& =-\frac{1}{2 \pi} \int_{\gamma} \mu(\sigma) \frac{K_{1}(s, \sigma)}{|s-\sigma|^{1-\lambda / 4}} d \sigma-\frac{1}{2 \pi} \int_{\gamma} \mu(\sigma) K_{2}(s, \sigma) d \sigma .
\end{aligned}
$$

The validity of differentiation under the integral can be proved in the same way as at the end of [9, Section 1.6] (Fubini theorem on change of integration order is used). Taking into account the obtained representation for $d I_{1}(s) / d s$ and applying results of [7, Section 51.1], we obtain that $d I_{1}(s) / d s \in C^{0, \lambda / 4}[a, b]$. Lemma is proved.

\section{Existence of A Classical Solution}

We will construct the solution to the problem $\mathbf{D}_{1}$ in assumption that $F^{+}(s), F^{-}(s) \in C^{1, \lambda}\left(\Gamma^{1}\right)$, $\lambda \in(0,1], F(s) \in C^{0}\left(\Gamma^{2}\right)$. Note that we do not require compatibility conditions at the tips of the cracks, that is, we we do not require that $F^{+}(d)=F^{-}(d)$ for any $x(d) \in X$. We will look for a solution to the problem $\mathbf{D}_{1}$ in the form

$$
u(x)=-w\left[F^{+}-F^{-}\right](x)+v(x)
$$

where

$$
w\left[F^{+}-F^{-}\right](x)=-\frac{1}{2 \pi} \int_{\Gamma^{1}}\left(F^{+}(\sigma)-F^{-}(\sigma)\right) \frac{\partial}{\partial \mathbf{n}_{y}} \ln |x-y(\sigma)| d \sigma
$$


is the double layer potential. The potential $w\left[F^{+}-F^{-}\right](x)$ satisfies the Laplace equation (2.7)

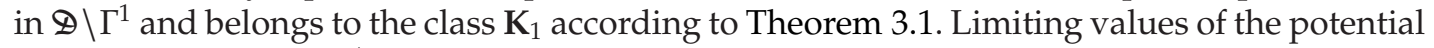
$w\left[F^{+}-F^{-}\right](x)$ on $\left(\Gamma^{1}\right)^{ \pm}$are given by the formula

$$
\left.w\left[F^{+}-F^{-}\right](x)\right|_{x(s) \in\left(\Gamma^{1}\right)^{ \pm}}=\mp \frac{F^{+}(s)-F^{-}(s)}{2}+w\left[F^{+}-F^{-}\right](x(s)),
$$

where $w\left[F^{+}-F^{-}\right](x(s))$ is the direct value of the potential on $\Gamma^{1}$.

The function $v(x)$ in (4.1) must be a solution to the following problem.

\section{Problem D}

Find a function $v(x) \in C^{0}(\bar{\Phi}) \cap C^{2}\left(\Phi \backslash \Gamma^{1}\right)$, which obeys the Laplace equation (2.7) in the domain $\Phi \backslash \Gamma^{1}$ and satisfies the boundary conditions

$$
\begin{gathered}
\left.v(x)\right|_{x(s) \in \Gamma^{1}}=\frac{F^{+}(s)+F^{-}(s)}{2}+w\left[F^{+}-F^{-}\right](x(s))=f(s), \\
\left.v(x)\right|_{x(s) \in \Gamma^{2}}=F(s)+w\left[F^{+}-F^{-}\right](x(s))=f(s) .
\end{gathered}
$$

If $x(s) \in \Gamma^{1}$, then $w\left[F^{+}-F^{-}\right](x(s))$ is the direct value of the potential on $\Gamma^{1}$.

If $\Phi$ is an exterior domain, then we add the following condition at infinity:

$$
|v(x)| \leq \text { const }, \quad|x|=\sqrt{x_{1}^{2}+x_{2}^{2}} \longrightarrow \infty .
$$

All conditions of the problem $\mathbf{D}$ have to be satisfied in a classical sense. Obviously, $w\left[F^{+}-F^{-}\right](x(s)) \in C^{0}\left(\Gamma^{2}\right)$. It follows from Lemma 3.3 that $w\left[F^{+}-F^{-}\right](x(s)) \in C^{1, \lambda / 4}\left(\Gamma^{1}\right)$ (here by $w\left[F^{+}-F^{-}\right](x(s))$ we mean the direct value of the potential on $\left.\Gamma^{1}\right)$. So, $f(s) \in C^{1, \lambda / 4}\left(\Gamma^{1}\right)$ and $f(s) \in C^{0}\left(\Gamma^{2}\right)$.

We will look for the function $v(x)$ in the smoothness class $\mathbf{K}$.

We say that the function $v(x)$ belongs to the smoothness class $\mathbf{K}$ if

(1) $v(x) \in C^{0}(\bar{\Phi}) \cap C^{2}\left(\Phi \backslash \Gamma^{1}\right), \quad \nabla v \in C^{0}\left(\overline{\Phi \backslash \Gamma^{1}} \backslash \Gamma^{2} \backslash X\right)$, where $X$ is a pointset consisting of the endpoints of $\Gamma^{1}$.

(2) in a neighbourhood of any point $x(d) \in X$ for some constants $\mathcal{C}>0, \delta>-1$ the inequality $|\nabla v| \leq \mathcal{C}|x-x(d)|^{\delta}$ holds, where $x \rightarrow x(d)$ and $d=a_{n}^{1}$ or $d=b_{n}^{1}$ $n=1, \ldots, N_{1}$.

The definition of the functional class $C^{0}\left(\overline{\boldsymbol{\Phi} \backslash \Gamma^{1}} \backslash \Gamma^{2} \backslash X\right)$ is given in the remark to the definition of the smoothness class $\mathbf{K}_{1}$. Clearly, $\mathbf{K} \subset \mathbf{K}_{1}$, that is, if $v(x) \in \mathbf{K}$, then $v(x) \in \mathbf{K}_{1}$.

It can be verified directly that if $v(x)$ is a solution to the problem $\mathbf{D}$ in the class $\mathbf{K}$, then the function (4.1) is a solution to the problem $\mathbf{D}_{1}$.

Theorem 4.1. Let $\Gamma \in C^{2, \lambda / 4}, f(s) \in C^{1, \lambda / 4}\left(\Gamma^{1}\right), \lambda \in(0,1], f(s) \in C^{0}\left(\Gamma^{2}\right)$. Then the solution to the problem $\mathbf{D}$ in the smoothness class $\mathbf{K}$ exists and is unique. 
Theorem 4.1 has been proved in the following papers:

(1) in $[2,3]$, if $\Phi$ is an interior domain;

(2) in [4], if $\Phi$ is an exterior domain and $\Gamma^{2} \neq \emptyset$;

(3) in $[5,6]$, if $\Gamma^{2}=\emptyset$ and so $\Phi=R^{2}$ is an exterior domain.

In all these papers, the integral representations for the solution to the problem $\mathbf{D}$ in the class $\mathbf{K}$ are obtained in the form of potentials, densities in which are defined from the uniquely solvable Fredholm integroalgebraic equations of the second kind and index zero. Uniqueness of a solution to the problem $\mathbf{D}$ is proved either by the maximum principle or by the method of energy (integral) identities. In the latter case, we take into account that a solution to the problem belongs to the class $\mathbf{K}$. Note that the problem $\mathbf{D}$ is a particular case of more general boundary value problems studied in [3-6].

Note that conditions of Theorem 4.1 hold if $\Gamma \in C^{2, \lambda}, F^{+}(s) \in C^{1, \lambda}\left(\Gamma^{1}\right), F^{-}(s) \in$ $C^{1, \lambda}\left(\Gamma^{1}\right), \lambda \in(0,1], F(s) \in C^{0}\left(\Gamma^{2}\right)$. From Theorems 3.1 and 4.1 we obtain the solvability of the problem $\mathbf{D}_{1}$.

Theorem 4.2. Let $\Gamma \in C^{2, \lambda}, F^{+}(s) \in C^{1, \lambda}\left(\Gamma^{1}\right), F^{-}(s) \in C^{1, \lambda}\left(\Gamma^{1}\right), \lambda \in(0,1], F(s) \in C^{0}\left(\Gamma^{2}\right)$. Then a solution to the problem $\mathbf{D}_{1}$ exists and is given by the formula (4.1), where $v(x)$ is a unique solution to the problem $\mathbf{D}$ in the class $\mathbf{K}$ ensured by Theorem 4.1.

Uniqueness of a solution to the problem $\mathbf{D}_{1}$ follows from Theorem 2.2. In fact, the solution to the problem $\mathbf{D}_{1}$ found in Theorem 4.2 is a classical solution. Let us discuss, under which conditions this solution to the problem $\mathbf{D}_{1}$ is not a weak solution.

\section{Nonexistence of a Weak Solution}

Let $u(x)$ be a solution to the problem $\mathrm{D}_{1}$ defined in Theorem 4.2 by the formula (4.1). Consider a disc $S(d, \epsilon)$ with the center in the point $x(d) \in X$ and of radius $\epsilon>0\left(d=a_{n}^{1}\right.$ or $\left.d=b_{n}^{1}, n=1, \ldots, N_{1}\right)$. In doing so, $\epsilon$ is a fixed positive number, which can be taken small enough. Since $v(x) \in \mathbf{K}$, we have $v(x) \in L_{2}(S(d, \epsilon))$ and $|\nabla v(x)| \in L_{2}(S(d, \epsilon))$ (this follows from the definition of the smoothness class $\mathbf{K})$. Let $x \in S(d, \epsilon)$ and $x \notin \Gamma^{1}$. It follows from (4.1) that $|\nabla w[\mu](x)| \leq|\nabla u(x)|+|\nabla v(x)|$, whence

$$
\begin{aligned}
|\nabla w[\mu](x)|^{2} & \leq|\nabla u(x)|^{2}+|\nabla v(x)|^{2}+2|\nabla u(x)| \cdot|\nabla v(x)| \\
& \leq 2\left(|\nabla u(x)|^{2}+|\nabla v(x)|^{2}\right),
\end{aligned}
$$

since $2|\nabla u(x)| \cdot|\nabla v(x)| \leq|\nabla u(x)|^{2}+|\nabla v(x)|^{2}$. Assume that $|\nabla u(x)|$ belongs to $L_{2}(S(d, \epsilon))$, then, integrating this inequality over $S(d, \epsilon)$, we obtain $\left.\|\nabla w\|^{2}\right|_{L_{2}(S(d, e))} \leq 2\left(\left.\|\nabla u\|^{2}\right|_{L_{2}(S(d, e))}+\right.$ $\left.\left.\|\nabla v\|^{2}\right|_{L_{2}(S(d, e))}\right)$. Consequently, if $|\nabla u(x)| \in L_{2}(S(d, \epsilon))$, then $|\nabla w| \in L_{2}(S(d, \epsilon))$. However, according to Theorem 3.1, if $F^{+}(d)-F^{-}(d) \neq 0$, then $|\nabla w|$ does not belong to $L_{2}(S(d, \epsilon)$ ). Therefore, if $F^{+}(d) \neq F^{-}(d)$, then our assumption that $|\nabla u| \in L_{2}(S(d, \epsilon))$ does not hold, that is, $|\nabla u| \notin L_{2}(S(d, \epsilon))$. Thus, if among numbers $a_{1}^{1}, \ldots, a_{N_{1}}^{1}, b_{1}^{1}, \ldots, b_{N_{1}}^{1}$ there exists such a number $d$ that $F^{+}(d) \neq F^{-}(d)$, then for some $\epsilon>0$, we have $|\nabla u| \notin L_{2}(S(d, \epsilon))=L_{2}\left(S(d, \epsilon) \backslash \Gamma^{1}\right)$, so $u \notin H^{1}\left(S(d, \epsilon) \backslash \Gamma^{1}\right)$, where $H^{1}$ is a Sobolev space of functions from $L_{2}$, which have generalized derivatives from $L_{2}$. We have proved the following theorem. 
Theorem 5.1. Let conditions of Theorem 4.2 hold and among numbers $a_{1}^{1}, \ldots, a_{N_{1}}^{1}, b_{1}^{1}, \ldots, b_{N_{1}}^{1}$ there exists such a number $d$, that $F^{+}(d) \neq F^{-}(d)$ (i.e., compatibility condition does not hold at the tip $x(d) \in X)$. Then the solution to the problem $\mathbf{D}_{1}$, ensured by Theorem 4.2, does not belong to $H^{1}\left(S(d, \epsilon) \backslash \Gamma^{1}\right)$ for some $\epsilon>0$, whence it follows that it does not belong to $H_{\mathrm{loc}}^{1}\left(\Phi \backslash \Gamma^{1}\right)$. Here $S(d, \epsilon)$ is a disc of a radius $\epsilon$ with the center in the point $x(d) \in X$.

By $H_{\text {loc }}^{1}\left(\boldsymbol{\Phi} \backslash \Gamma^{1}\right)$ we denote a class of functions, which belong to $H^{1}$ on any bounded subdomain of $\Phi \backslash \Gamma^{1}$. If conditions of Theorem 5.1 hold, then the unique solution to the problem $\mathbf{D}_{1}$, constructed in Theorem 4.2, does not belong to $H_{\mathrm{loc}}^{1}\left(\boldsymbol{\Phi} \backslash \Gamma^{1}\right)$, and so it is not a weak solution. We arrive to the following corollary.

Corollary 5.2. Let conditions of Theorem 5.1 hold, then a weak solution to the problem $\mathbf{D}_{1}$ in the class of functions $H_{\mathrm{loc}}^{1}\left(\Phi \backslash \Gamma^{1}\right)$ does not exist.

Remark 5.3. It should be stressed that even if closed curves and cracks are very smooth and if boundary data is very smooth as well, but if there exists a tip of the crack, where the compatibility condition does not hold, then a weak solution of the problem $\mathbf{D}_{1}$ in the class of functions $H_{\mathrm{loc}}^{1}\left(\boldsymbol{\Phi} \backslash \Gamma^{1}\right)$ does not exist.

Remark 5.4. Even if the number $d$, mentioned in Theorem 5.1, does not exist, then the solution $u(x)$ to the problem $\mathrm{D}_{1}$, ensured by Theorem 4.2 , may not be a weak solution to the problem $\mathbf{D}_{1}$. The Hadamard example of a nonexistence of a weak solution to a harmonic Dirichlet problem in a disc with continuous boundary data is given in [11, Section 12.5] (the classical solution exists in this example).

Clearly, $L_{2}\left(\boldsymbol{\Phi} \backslash \Gamma^{1}\right)=L_{2}(\boldsymbol{\Phi})$, since $\Gamma^{1}$ is a set of zero measure.

\section{Singularities of the Gradient of the Solution at the Endpoints of the Cracks}

It follows from Theorems 4.2 and 4.1 that the gradient of the solution of problem $\mathrm{D}_{1}$ given by formula (4.1) can be unbounded at the endpoints of the cracks $\Gamma^{1}$.

Let $v(x)$ be a solution of the Problem $\mathrm{D}$ ensured by Theorem 4.1. Let $u(x)$ be a solution of the Problem $\mathbf{D}_{1}$ ensured by Theorem 4.2 and given by formula (4.1). Let $x(d) \in X$ be one of the endpoints of $\Gamma^{1}$. In the neighbourhood of $x(d)$, we introduce the system of polar coordinates

$$
x_{1}=x_{1}(d)+|x-x(d)| \cos \psi(x, x(d)), \quad x_{2}=x_{2}(d)+|x-x(d)| \sin \psi(x, x(d)) .
$$

We assume that $\psi(x, x(d)) \in(\alpha(d), \alpha(d)+2 \pi)$ if $d=a_{n}^{1}$, and $\psi(x, x(d)) \in(\alpha(d)-\pi, \alpha(d)+\pi)$ if $d=b_{n}^{1}$. We recall that $\alpha(s)$ is the angle between the direction of the $O x_{1}$ axis and the tangent vector $\tau_{x}$ to $\Gamma^{1}$ at the point $x(s)$.

Hence, $\alpha(d)=\alpha\left(a_{n}^{1}+0\right)$ if $d=a_{n}^{1}$, and $\alpha(d)=\alpha\left(b_{n}^{1}-0\right)$ if $d=b_{n}^{1}$.

Thus, the angle $\psi(x, x(d))$ varies continuously in the neighbourhood of the endpoint $x(d)$, cut along $\Gamma^{1}$.

Let $\mu(s)$ be a solution of the integral equation ensured by Theorem 4 in [2] or by Theorem 4.4 in [3] or by Theorem 4 in [4]. The integral representation for the solution $v(x)$ 
of the Problem $\mathbf{D}$ is constructed in [2-4] on the basis of the function $\mu(s)$ that is a solution of the certain integral equation.

Alternatively, one can assume that $\mu(s)$ is an element of a solution to equations ensured by Corollary 3.2 in [5] or by Theorem 4 in [6]. The solution $v(x)$ of the Problem $\mathbf{D}$ is constructed in $[5,6]$ with the help of the function $\mu(s)$, which is an element of a solution to certain equations.

We will use the notation $\mu_{1}(s)=\mu(s)|s-d|^{1 / 2}$, and put $\mu_{1}(d)=\mu_{1}\left(a_{n}^{1}\right)=\mu_{1}\left(a_{n}^{1}+0\right)$ if $d=a_{n}^{1}$, and $\mu_{1}(d)=\mu_{1}\left(b_{n}^{1}\right)=\mu_{1}\left(b_{n}^{1}-0\right)$ if $d=b_{n}^{1}$.

At first, we study the behaviour of the gradient of a solution $v(x)$ of the problem $\mathbf{D}$ at the tips of the cracks. Using the representation of the derivatives of harmonic potentials in terms of Cauchy type integrals (see [10]) and using the properties of these integrals near the endpoints of the integration line, presented in [7], we can prove the following assertion.

Theorem 6.1. Let $v(x)$ be a solution of the problem $\mathbf{D}$ ensured by Theorem 4.1. Let $x(d)$ be an arbitrary endpoint of the cracks $\Gamma^{1}$, that is, $x(d) \in X$ and $d=a_{n}^{1}$ or $d=b_{n}^{1}$ for some $n=1, \ldots, N_{1}$. Then the derivatives of the solution of the problem $\mathbf{D}$ in the neighbourhood of $x(d)$ have the following asymptotic behaviour.

If $d=a_{n}^{1}$, then

$$
\begin{gathered}
\frac{\partial}{\partial x_{1}} v(x)=\frac{\mu_{1}\left(a_{n}^{1}\right)}{2\left|x-x\left(a_{n}^{1}\right)\right|^{1 / 2}} \sin \left(\frac{\psi\left(x, x\left(a_{n}^{1}\right)\right)+\alpha\left(a_{n}^{1}\right)}{2}\right)+O(1), \\
\frac{\partial}{\partial x_{2}} v(x)=-\frac{\mu_{1}\left(a_{n}^{1}\right)}{2\left|x-x\left(a_{n}^{1}\right)\right|^{1 / 2}} \cos \left(\frac{\psi\left(x, x\left(a_{n}^{1}\right)\right)+\alpha\left(a_{n}^{1}\right)}{2}\right)+O(1),
\end{gathered}
$$

If $d=b_{n}^{1}$, then

$$
\begin{aligned}
& \frac{\partial}{\partial x_{1}} v(x)=-\frac{\mu_{1}\left(b_{n}^{1}\right)}{2\left|x-x\left(b_{n}^{1}\right)\right|^{1 / 2}} \cos \left(\frac{\psi\left(x, x\left(b_{n}^{1}\right)\right)+\alpha\left(b_{n}^{1}\right)}{2}\right)+O(1), \\
& \frac{\partial}{\partial x_{2}} v(x)=-\frac{\mu_{1}\left(b_{n}^{1}\right)}{2\left|x-x\left(b_{n}^{1}\right)\right|^{1 / 2}} \sin \left(\frac{\psi\left(x, x\left(b_{n}^{1}\right)\right)+\alpha\left(b_{n}^{1}\right)}{2}\right)+O(1) .
\end{aligned}
$$

By $O(1)$ we denote functions which are continuous at the endpoint $x(d)$. Moreover, the functions denoted as $O(1)$ are continuous in the neighbourhood of the endpoint $x(d)$ cut along $\Gamma^{1}$ and are continuously extensible to $\left(\Gamma^{1}\right)^{+}$and to $\left(\Gamma^{1}\right)^{-}$from this neighbourhood.

The formulas of the theorem demonstrate the following curious fact. In the general case, the derivatives of the solution of problem $\mathbf{D}$ near the endpoint $x(d)$ of cracks $\Gamma^{1}$ behave as $O\left(|x-x(d)|^{-1 / 2}\right)$. However, if $\mu_{1}(d)=0$, then $\nabla v(x)$ is bounded and even continuous at the endpoint $x(d) \in X$.

On the basis of Theorem 3.1, Theorem 6.1 and formula (4.1), we may study the behaviour of the gradient of a solution $u(x)$ of the problem $\mathbf{D}_{1}$ at the tips of the cracks. Using notations introduced above and notations from Section 3, we arrive at the following assertion. 
Theorem 6.2. Let $u(x)$ be a solution of the problem $\mathbf{D}_{1}$ ensured by Theorem 4.2. Let $x(d)$ be an arbitrary endpoint of the cracks $\Gamma^{1}$, that is, $x(d) \in X$ and $d=a_{n}^{1}$ or $d=b_{n}^{1}$ for some $n=1, \ldots, N_{1}$. Then the derivatives of the solution of the problem $\mathbf{D}_{1}$ in the neighbourhood of $x(d)$ have the following asymptotic behaviour.

$$
\text { If } d=a_{n}^{1} \text {, then }
$$

$$
\begin{aligned}
\frac{\partial}{\partial x_{1}} u(x)= & \frac{1}{2 \pi} \frac{F^{+}\left(a_{n}^{1}\right)-F^{-}\left(a_{n}^{1}\right)}{\left|x-x\left(a_{n}^{1}\right)\right|} \sin \psi\left(x, x\left(a_{n}^{1}\right)\right) \\
& +\frac{\mu_{1}\left(a_{n}^{1}\right)}{2\left|x-x\left(a_{n}^{1}\right)\right|^{1 / 2}} \sin \left(\frac{\psi\left(x, x\left(a_{n}^{1}\right)\right)+\alpha\left(a_{n}^{1}\right)}{2}\right) \\
& +\frac{F^{\prime+}\left(a_{n}^{1}\right)-F^{\prime-}\left(a_{n}^{1}\right)}{2 \pi}\left(\sin \alpha\left(a_{n}^{1}\right) \ln \left|x-x\left(a_{n}^{1}\right)\right|-\psi\left(x, x\left(a_{n}^{1}\right)\right) \cos \alpha\left(a_{n}^{1}\right)\right)+O(1), \\
\frac{\partial}{\partial x_{2}} u(x)= & -\frac{1}{2 \pi} \frac{F^{+}\left(a_{n}^{1}\right)-F^{-}\left(a_{n}^{1}\right)}{\left|x-x\left(a_{n}^{1}\right)\right|} \cos \psi\left(x, x\left(a_{n}^{1}\right)\right) \\
& -\frac{\mu_{1}\left(a_{n}^{1}\right)}{2\left|x-x\left(a_{n}^{1}\right)\right|^{1 / 2}} \cos \left(\frac{\psi\left(x, x\left(a_{n}^{1}\right)\right)+\alpha\left(a_{n}^{1}\right)}{2}\right) \\
& -\frac{F^{\prime+}\left(a_{n}^{1}\right)-F^{\prime-}\left(a_{n}^{1}\right)}{2 \pi}\left(\cos \alpha\left(a_{n}^{1}\right) \ln \left|x-x\left(a_{n}^{1}\right)\right|+\psi\left(x, x\left(a_{n}^{1}\right)\right) \sin \alpha\left(a_{n}^{1}\right)\right)+O(1) .
\end{aligned}
$$

If $d=b_{n}^{1}$, then

$$
\begin{aligned}
\frac{\partial}{\partial x_{1}} u(x)= & -\frac{1}{2 \pi} \frac{F^{+}\left(b_{n}^{1}\right)-F^{-}\left(b_{n}^{1}\right)}{\left|x-x\left(b_{n}^{1}\right)\right|} \sin \psi\left(x, x\left(b_{n}^{1}\right)\right) \\
& -\frac{\mu_{1}\left(b_{n}^{1}\right)}{2\left|x-x\left(b_{n}^{1}\right)\right|^{1 / 2}} \cos \left(\frac{\psi\left(x, x\left(b_{n}^{1}\right)\right)+\alpha\left(b_{n}^{1}\right)}{2}\right) \\
& -\frac{F^{\prime+}\left(b_{n}^{1}\right)-F^{\prime^{-}}\left(b_{n}^{1}\right)}{2 \pi}\left(\sin \alpha\left(b_{n}^{1}\right) \ln \left|x-x\left(b_{n}^{1}\right)\right|-\psi\left(x, x\left(b_{n}^{1}\right)\right) \cos \alpha\left(b_{n}^{1}\right)\right)+O(1), \\
\frac{\partial}{\partial x_{2}} u(x)= & \frac{1}{2 \pi} \frac{F^{+}\left(b_{n}^{1}\right)-F^{-}\left(b_{n}^{1}\right)}{|x-x(d)|} \cos \psi\left(x, x\left(b_{n}^{1}\right)\right) \\
& -\frac{\mu_{1}\left(b_{n}^{1}\right)}{2\left|x-x\left(b_{n}^{1}\right)\right|^{1 / 2}} \sin \left(\frac{\psi\left(x, x\left(b_{n}^{1}\right)\right)+\alpha\left(b_{n}^{1}\right)}{2}\right) \\
& +\frac{F^{\prime+}\left(b_{n}^{1}\right)-F^{\prime-}\left(b_{n}^{1}\right)}{2 \pi}\left(\cos \alpha\left(b_{n}^{1}\right) \ln \left|x-x\left(b_{n}^{1}\right)\right|+\psi\left(x, x\left(b_{n}^{1}\right)\right) \sin \alpha\left(b_{n}^{1}\right)\right)+O(1) .
\end{aligned}
$$


$B y O(1)$ we denote functions which are continuous at the endpoint $x(d)$. Moreover, the functions denoted as $O(1)$ are continuous in the neighbourhood of the endpoint $x(d)$ cut along $\Gamma^{1}$ and are continuously extensible to $\left(\Gamma^{1}\right)^{+}$and to $\left(\Gamma^{1}\right)^{-}$from this neighbourhood.

In the formulation of the theorem we use the notation $F^{ \pm}(s)=d F^{ \pm}(s) / d s$. The formulas of the theorem demonstrate the following curious fact. In the general case, the derivatives of the solution of problem $\mathbf{D}_{1}$ near the endpoint $x(d)$ of cracks $\Gamma^{1}$ behave as

$$
O\left(\frac{1}{|x-x(d)|}\right)+O\left(\frac{1}{|x-x(d)|^{1 / 2}}\right)+O\left(\ln \frac{1}{|x-x(d)|}\right)
$$

However, if $F^{+}(d)-F^{-}(d)=\mu_{1}(d)={F^{\prime}}^{+}(d)-{F^{\prime}}^{-}(d)=0$, then $\nabla u(x)$ is bounded and even continuous at the endpoint $x(d) \in X$.

\section{References}

[1] I. K. Lifanov, Method of Singular Integral Equations and Discrete Vortices, VSP, Zeist, The Netherlands, 1996.

[2] P. A. Krutitskii, "The integral representation for a solution of the 2-D Dirichlet problem with boundary data on closed and open curves," Mathematika, vol. 47, no. 1-2, pp. 339-354, 2000.

[3] P. A. Krutitskii, "The Dirichlet problem for the 2-D Laplace equation in a multiply connected domain with cuts," Proceedings of the Edinburgh Mathematical Society, Series 2, vol. 43, no. 2, pp. 325-341, 2000.

[4] P. A. Krutitskii, "The 2-dimensional Dirichlet problem in an external domain with cuts," Zeitschrift für Analysis und ihre Anwendungen, vol. 17, no. 2, pp. 361-378, 1998.

[5] P. A. Krutitskii, "A mixed problem for the Laplace equation outside cuts on the plane," Differential Equations, vol. 33, no. 9, pp. 1184-1193, 1997.

[6] P. A. Krutitskii, "The mixed harmonic problem in an exterior cracked domain with Dirichlet condition on cracks," Computers and Mathematics with Applications, vol. 50, no. 5-6, pp. 769-782, 2005.

[7] N. I. Muskhelishvili, Singular Integral Equations, Nauka, Moscow, Russia, 1968 (in Russian). English translation: Noordhoff, Groningen, The Netherlands, 1972.

[8] D. Gilbarg and N. S. Trudinger, Elliptic Partial Differential Equations of Second Order, Springer, New York, NY, USA, 1977.

[9] V.S. Vladimirov, Equations of Mathematical Physics, Nauka, Moscow, Russia, 1981 (in Russian). English translation: Mir Publishers, Moscow, Russia, 1984.

[10] P. A. Krutitskii, "Dirichlet's problem for the Helmholtz equation outside cuts in a plane," Computational Mathematics and Mathematical Physics, vol. 34, no. 8-9, pp. 1073-1090, 1994.

[11] S. L. Sobolev, Some Applications of Functional Analysis to Mathematical Physics, Nauka, Moscow, Russia, 1988 (in Russian). 


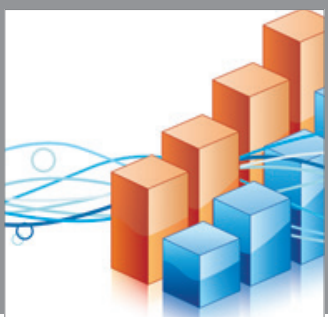

Advances in

Operations Research

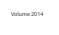

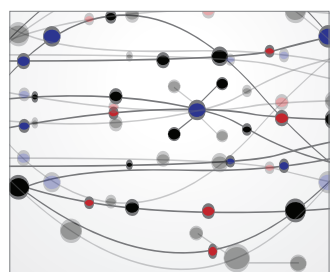

\section{The Scientific} World Journal
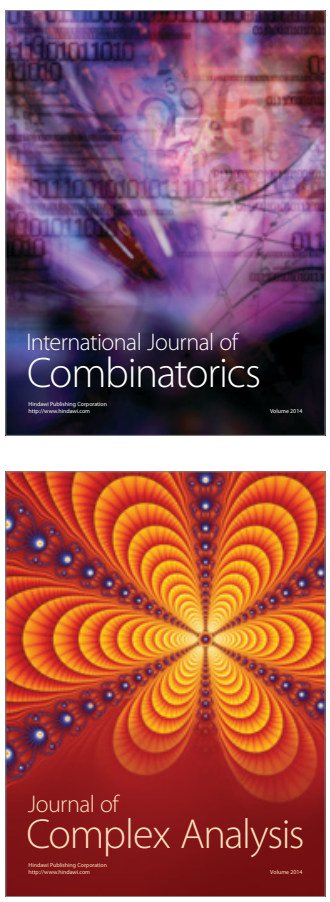

International Journal of

Mathematics and

Mathematical

Sciences
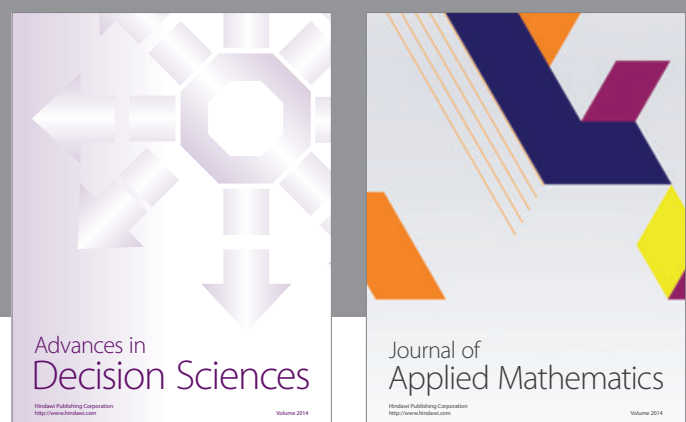

Journal of

Applied Mathematics
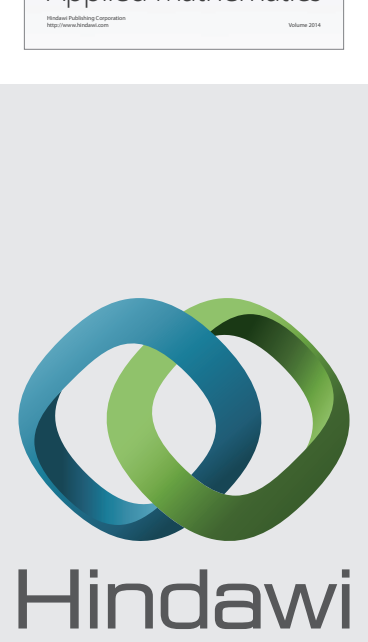

Submit your manuscripts at http://www.hindawi.com
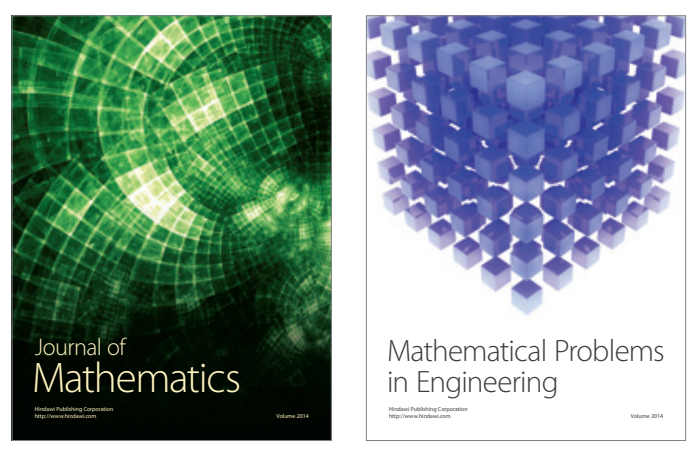

Mathematical Problems in Engineering
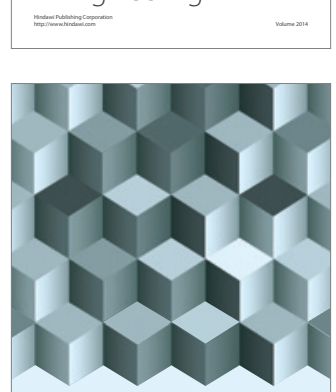

Journal of

Function Spaces
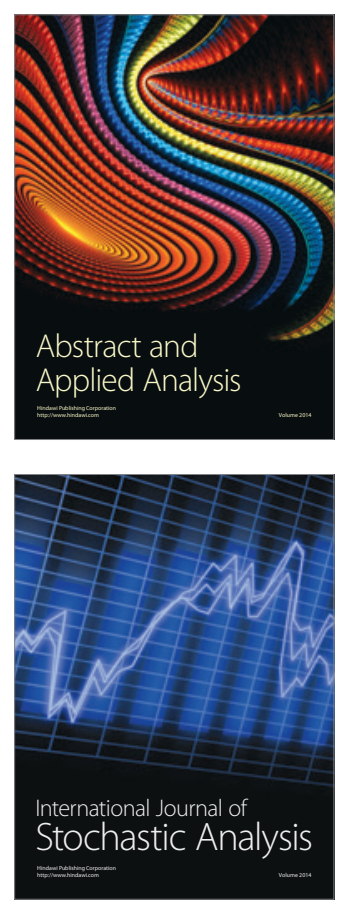

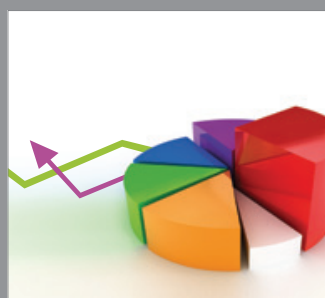

ournal of

Probability and Statistics

Promensencen
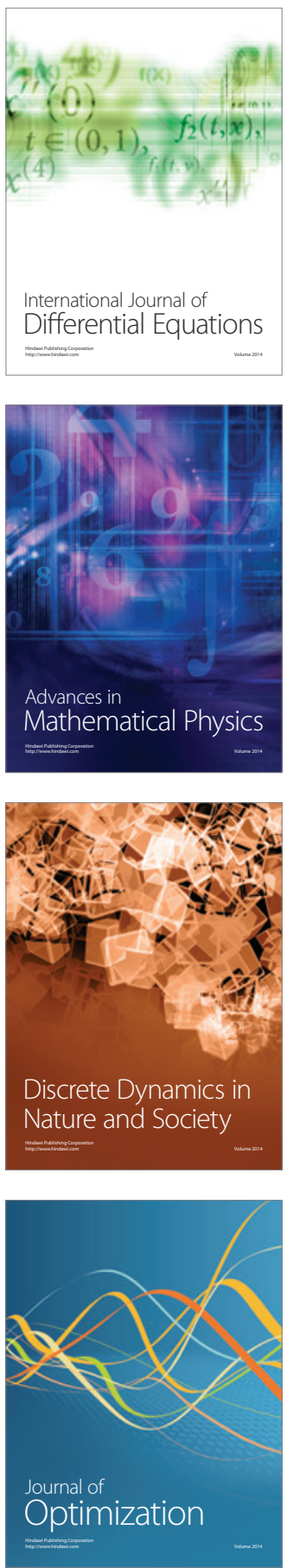